\title{
ON WEAKER FORMS OF COMPACTNESS LINDELÖFNESS AND COUNTABLE COMPACTNESS
}

D. BABOOLAL, J. BACKHOUSE and R.G. ORI

\author{
Department of Mathematics \\ University of Durban-Westville \\ Private Bag X54001 \\ Durban 4000 \\ South Africa
}

(Received July 6, 1987 and in revised form August 9, 1988)

\begin{abstract}
A theory of e-countable compactness and e-Lindelöfness which are weaker than the concepts of countable compactness and Lindelofness respectively is developed. Amongst other results we show that an e-countably compact space is pseudocompact, and an example of a space which is pseudocompact but not e-countably compact.. with respect to any dense set is presented. We also show that every eLindelof metric space is separable.
\end{abstract}

KEY WORDS AND PHRASES. e-compact, e-countably compact, e-Lindelof, pseudocompact, separable.

1980 AMS SUBJECT CLASSIFICATION CODES. 54A05, 54D30, $54 \mathrm{G} 20$.

1. INTRODUCTION.

Using the terminology and notation in [3], it is known that a topological space X is compact iff its enlargement *X contains only near-standard points, and that a subset $A$ of a regular Hausdorff space is relatively compact iff $A$ contains only near standard points. Hechler [1] wanted to know what this condition implied topologically in not necessarily regular spaces. He was led to the notion of what he called 'ecompactness' which is weaker than the notion of compactness. It is the purpose of this paper to develop a theory on the analogous concepts of e-countable compactness and e-Lindelofness in the spirit of Hechler's study of e-compactness [1]. In particular we extend the we1l-known result that a countably compact space is pseudocompact to an e-countably compact space is pseudocompact. We also show that the Lindelof condition in the theorem that every Lindelof metric space is separable can be weakened to e-Lindelof. 
2. PRELIMINARIES.

We begin by recalling Hechler's definition of e-compactness and e-regularity.

Let $D$ be a dense subspace of a lopological space $X$.

DEFINITION 2.1. ( $[1]):$ (a) $X$ is said to be e-compact with respect to $D$ if each open cover of $X$ contains a fintte subcollection that covers $D$. (b) $X$ is e-regular with respect to a dense subset $D$ if for each closed $F \subset x$ and each $p / F$ there exisi disjoint open sets $U$ and $V$ such that $P$ : $U$ and $F \cap D \subset V$. By analogy we introdice the following:

DEFINITION 2.2. $X$ is e-countably conpact with respect to $D$ if every couniable open cover of $X$ has a finite subcollection covering $D$.

DEFINITLON 2.3. $X$ is e-Lindelöf with respect to $D$ if every open cover of $X$ ha:s 1 countable subcollection covering $D$.

Recall Hechler's extension of the topology $T$ for $X$ :

Let $E$ be a family of subsets of $X$, and let $T(E)=\{U-F \mid U: T$ and $F$ is a subset of a finile union of members of $E$. By $X(E)$ we shall mean $X$ with the extended topology $T(E)$. Hechler used the construction of the extended topology $T(E)$ to provide examples of spaces which are e-compact but not compact (see [1] p. 223).

\section{RESULTS.}

The following two theorems are analogous to the corresponding theorems on ecompactness (see [1]).

THEOREM 3.1. Let $X$ be Lindelof. Then $X(E)$ is e-lindelof iff there exists a dense set $D$ (with respect to $X(E)$ ) such that for every $E \varepsilon E$, $E \cap D$ is countable.

PROOF. Suppose $X(E)$ is e-Lindelof with respect to a dense set $D$. Assume for some $E \in E, E \cap D$ is uncountable. Then $\{X-(E-\{x\}) \mid x E E\}$ is an open cover of $X$ having no countable subcover of $D$, contrary to $X(E)$ being e-lindelof. Thus $E \cap D$ is countable for all $E \in E$.

Conversely suppose $E \cap D$ is countable for all $E \varepsilon E$ and $D$ is dense with respect to $X(E)$. Suppose $\left\{U_{\alpha}-F_{i \alpha}: \alpha \varepsilon \Lambda\right\}$ is an open cover of $X$. Then $\left\{U_{\alpha}: \alpha \varepsilon \Lambda\right\}$ covers $X$, and as $X$ is Lindelof, there is a countable subcollection $\left\{U_{\alpha_{i}}\right\}_{i=1}^{\infty}$, say, covering $x$. Now $\left\{U_{\alpha_{i_{\infty}}}-F_{\alpha_{j}}\right\}_{i=1}^{\infty}$ will cover all except at most countably many points $\left\{x_{j}\right\}_{j=1}^{\infty}$ of $D$. But $\left\{x_{j}\right\}_{j=1}^{\infty} \subset U g$, where 8 is a countable subcollection of $\left\{U_{\alpha}-F_{\alpha}: \alpha \varepsilon \Lambda\right\}$. Thus $\left\{U_{\alpha_{i}}-F_{\alpha_{1}}\right\}_{i=1}^{\infty} U B$ is a countable subcollection of $\left\{U_{\alpha}-F_{\alpha}: \alpha \varepsilon \Lambda\right\}$ covering $D$, showing that $X(E)$ is e-tindelof.

THEOREM 3.2. Let $X$ be countably compact. Then $X(E)$ is e-countably coinpact iff there exists a dense subset $D$ (with respect to $X(E)$ ) such that for every set $E E E$, $E \cap D$ is finite.

PROOF. Suppose $X(E)$ is e-countably compact with respect to D. As sume for some $E \in E, E \cap D$ is infinite. Choose an infinite sequence $\left\{x_{n}: n E \mathbb{N}\right\}$ in $E \cap D$ and let $F=\left\{x_{n}: n \in \mathbb{N}\right\}$. Then $\{X-(F-\{x\}): x \in F\}$ is a countable open cover of $X$ with no finite subcover of $D$, contrary to $X$ being e-countably compact. 
Conversely suppose $E \cap D$ is finite for all $E \varepsilon E$, and $D$ a dense set with respect to $X(E)$. Suppose $\left\{U_{i}-F_{i}\right\}_{i=1}^{\infty}$ is a countable open cover of $X(E)$. Then $\left\{U_{1}\right\}_{1=1}^{\infty}$ covers $X$, and as $x$ is countably compact there is a fintte subcover $\left\{U_{k_{1}}\right\}_{l=1}^{r}$ of $x$. Now $\left\{U_{k_{i}}-F_{k_{1}}\right\}^{r}$ covers all except at most finitely many points of $D,\left\{x_{j}\right\}_{j=1}^{t}$, say. But $\left\{x_{j}\right\}_{j=1}^{L} \subset U \&$ where $\&$ is a finite subcollection of $\left\{U_{1}-F_{1}\right\}_{1=1}^{\infty}$. Thus $\left\{U_{k_{i}}-F_{k_{1}}\right\}_{i=1}^{r} \cup \&$ is a finite subcollection of $\left\{U_{1}-F_{1}\right\}_{i=1}^{\infty}$ covering $D$, showing $X(E)$ is e-countably compact.

THEOREM 3.3. If $X$ is e-countably compact, then $X$ is pseudocompact.

PROOF. Suppose $X$ is e-countably compact with respect to $D$. Let $f$ be a continuous real valued function of $x$. Then if $G_{n}=\{x \in X|| f(x) \mid<n\},\left\{G_{n}: n \varepsilon \mathbb{I N}\right\}$ would be an open cover of $X$ having a finite subcover of $D$. Clearly then $D \subset G_{n}$ for some $n \varepsilon I N$. By continuity of $f$, we have $f(X) \subset[-n, n]$, showing $f$ is bounded. It is well known that a countably compact first countable Hausdorff space is regular. We have the following:

THEOREM 3.4. Every e-countably compact first countable Hausdorff space is eregular (with respect to a dense set $D$ ).

PROOF. Let $\mathrm{p} \neq \mathrm{F}, \mathrm{F}$ closed in $\mathrm{X}$. Let $B$ be a countable open neighbourhood base at p. Since $X$ is Hausdorff, for each $q \varepsilon F$ there exists open $G_{q}$ and $B_{q} \varepsilon B$ such that $G_{q} \cap B_{q}=\phi$. Let $B^{\prime}=\left\{B_{q}\right\}_{q} \in F$, which, being a subfamily of $B$, must be countable. For each $B \in B^{\prime}$ let $H_{B}=U\left\{G_{q} \mid G_{q} \cap B=\phi\right\}$. Then $\left\{H_{B} \mid B \varepsilon B^{\prime}\right\}$

is a countable famlly of open sets covering $F$ so that $\{X-F\} \cup\left\{H_{B} \mid B_{\varepsilon} B^{\prime}\right\}$ covers $X$. Since $X$ is e-countably compact there exists a finite family $B^{\prime \prime} \subset B^{\prime}$ such that $D \subset(X-F) \cup U\left\{H_{B}: B \varepsilon B^{\prime \prime}\right\}$. Thus $F \cap D \subset U\left\{H_{B}: B \in B^{\prime \prime}\right\}=V$. Let $U=\cap\left\{B: B \in B^{\prime \prime}\right\}$ which is open and contains $\mathrm{p}$. It is easily verified that $\mathrm{U} \cap \mathrm{V}=\phi \cdot$

We now show how separability relates to the generalizations of compactness and Lindelofness introduced above. It is we 11 known that every Lindelof metric space is separable. In fact the Lindelöf condition can be weakened to e-Lindelöness as the following result shows.

THEOREM 3.5. If $X$ is metric and e-Lindelö with respect to a dense set $D$ then $X$ is separable.

PROOF. For each $n \in \mathbb{I N}$ let $U_{n}=\{S(x, 1 / n) \mid x \varepsilon X\}$. Since $U_{n}$ is an open cover of $X$, there exists a countable set $F_{n} \subset X$ such that $D \subset U\left\{S(x, 1 / n): x \varepsilon F_{n}\right\}$. Let $\mathbf{F}=U\left\{\mathrm{~F}_{\mathrm{n}}: \mathrm{n} \varepsilon \mathbb{I N}\right\}$. Then $\mathrm{F}$ is countable. 
ClAIM. F is dense in $X$ : Let $y \in X$ be arbitrary, $\varepsilon>0$. Find $N$ such that $1 / N<\varepsilon$. Now $S(y, 1 / 2 N) \cap D \neq \phi$ so there exists $z \in D$ such that $d(y, z)<1 / 2 N$. Now $D \subset U\left\{S(x, 1 / 2 N): \times \varepsilon F_{2 N}\right\}$ implies that there exists $\times \varepsilon F_{2 N} \subset F$ such that $d(z, x)<1 / 2 N$. Thus $d(y, x)<d(y, z)+d(z, x)<1 / 2 N+1 / 2 N=1 / N<\varepsilon$.

Hence $S(y, \varepsilon) \cap F \neq \phi$, showing $F$ dense in $X$.

We then have:

THEOREM 3.6. For metric spaces the following a re equivalent

(a) $X$ is separable

(b) $X$ is 2 nd countable

(c) $X$ is Lindelö

(d) $X$ is e-Lindelö (with respect to any dense set)

Since every e-countably compact space is pseudocomact and every pse udocompact $\mathrm{T}_{4}$ space is countably compact we also have

THEOREM 3.7. For $T_{4}$ spaces, the following are equivalent

(a) $X$ is countably compact

(b) $X$ is e-countably compact (with respect to any dense set)

(c) $X$ is pseudocompact

\section{EXAMP LES.}

This is an example of a space which is pseudocompact but not e-countably compact with respect to any dense set.

Consider $\mathrm{z}^{+}$, the positive integers with the relatively prime topology, 1.e. with basis $B=\left\{U_{a}(b) \mid a, b \in Z^{+},(a, b)=1\right\}$ where $U_{a}(b)=\left\{b+n a \varepsilon Z^{+} \mid n \varepsilon Z\right\}$. We shall show

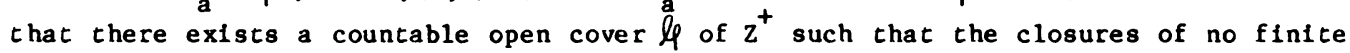
subcollection of $l$ covers $z^{+}$. This would then imply that $z^{+}$is not e-countably compact with respect to any dense set. Recall that $\mathrm{z}^{+}$is pseudocompact $([2], \mathrm{p} .83)$. Let then $l_{P}=\left\{U_{3}(1), U_{5}(2), U_{5}(3), U_{5}(4), U_{7}(5), U_{7}(6), U_{11}(7), U_{11}(8), \ldots\right\}$, and let $\varphi^{\prime}=\left\{U_{p_{1}}\left(\alpha_{1}\right)\right\}_{1=1}^{n}$ be any finite subcollection of $\varphi$.

Case 1: $U_{3}(1) \neq l \cdot$. Then let $m=p_{1} p_{2} \cdots p_{n}$ and note that

so that $\cup \bar{l}^{\prime} \neq \mathrm{z}^{+}$.

$$
U_{m}(m+1) \cap U_{P_{1}}\left(\alpha_{1}\right)=\emptyset \forall 1
$$

Ca se 2: $U_{3}(1) \varepsilon l^{\prime}$. If $U_{3}(1)$ is the only element in $\varphi^{\prime}$, then $\mathrm{U}_{3}(2) \cap \mathrm{U}_{3}(1)=\phi$, so that $\cup \overline{l_{q}} \neq \mathrm{z}^{+}$; otherwise let $\mathrm{m}=\mathrm{p}_{2} \mathrm{p}_{3} \cdots \mathrm{p}_{\mathrm{n}}$ where $\mathrm{p}_{1}=3$, and $\mathrm{p}_{1} \neq 3 \forall \mathrm{i}, 2<1<\mathrm{m}$.

Then

$$
U_{m}(m+1) \cap U_{p_{1}}\left(\alpha_{1}\right)=\phi \forall 1,2<1<n
$$

and $U_{3}(m+1) \cap U_{3}(1)=\phi\left(i n\right.$ the case where $3 /(m+1)$ so that $U_{m}(m+1) \cap U_{3}(m+1)$ is a neighbourhood of $\mathrm{m}+1$ meeting no member of $\zeta^{\prime}$. If $3 Y_{\mathrm{m}+1}$, let

$$
m^{\prime}=p_{0} m
$$


where $p_{0}$ is a prime $33 Y_{p_{0}}-1$ and $p_{0} \neq 3 . \quad$ Then $3 \chi_{p_{0}} m$ and $3 Y_{p_{0}} m+1$. Hence $U_{m^{\prime}}\left(m^{\prime}+1\right) \cap U_{3}\left(m^{\prime}+1\right)$ is the required neighbourhood of $m^{\prime}+1$ meeting no member of l. .

This example is motivated by the result that a Lindelöf countably compact space is compact. The analogous statement that an e-tindelöf, e-countably compact space is ecompact is not in general true as the following example shows:

Recall the Novak space (see [2] p. 134): Let $\mathrm{z}^{+}$denote the positive integers with the discrete topology and $S$ the Stone-Cech compactification $B Z^{+}$of $Z^{+}$. Let $F$ be the family of all countably infinite subsets of $S$, well-ordered by the least ordinal $\Gamma$ of cardinal $2^{c}=\operatorname{card}(S)$. Let $\left\{P_{A} \mid A \varepsilon F\right\}$ be a collection of subsets of $S$ such that card $\left(P_{A}\right)<2^{C}, P_{D} \subset P_{A}$ whenever $D<A$, and $\hat{f}\left(P_{A}\right) \cap P_{A}=\phi$ where $\hat{f}$ is the unique extension to $S$ of the continuous function $\mathrm{f}: \mathrm{Z}^{+}+\mathrm{Z}^{+}$which permutes each odd integer with its even successor, 1.e. $f(n)=n+(-1)^{n+1}$. Then we define $P=U\left\{P_{A} \mid A \varepsilon F\right\}$, and then define Novak's space by

$$
\mathrm{X}=\mathrm{P} \cup \mathrm{z}^{+} \text {. }
$$

Note that $c l_{X}\left(z^{+}\right)=X\left([2]\right.$ p. 135), hence $X$ is e-Lindelöf with respect to $Z^{+}$. Also $X$ is countably compact ( $[2]$ p. 135), but as $X$ is not compact, $X$ cannot be absolutely closed (as a regular absolutely closed space is compact). Thus $X$ is not e-compact with respect to any dense set.

\section{REFERENCES}

1. HECHLER, S.H., On a Notion of Weak Compactness in Non-Regular Spaces, Studies in Topology, (Edited by N.M. Stavrakas, K.R. Allen). Academlc Press, Inc., (1975), 215-237.

2. STEEN, L.A. and SEEBACH, J.A., Jr., Counterexamples in Topology (2nd Ed.) Springer-Verlag, New York, Heidelberg, Berl1n, (1978).

3. MACHOVER, M. and HIRSCHFIELD, Lectures on Non-Standard Analysis, Lecture Notes in Mathematics,94 Springer-Verlag, Berlin - New York, 1969. 


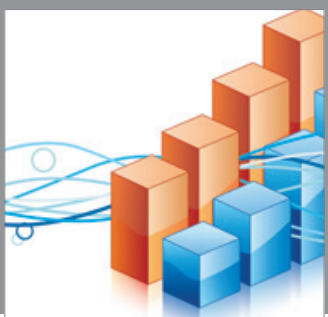

Advances in

Operations Research

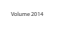

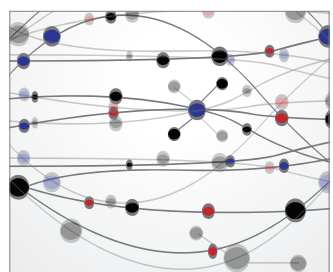

\section{The Scientific} World Journal
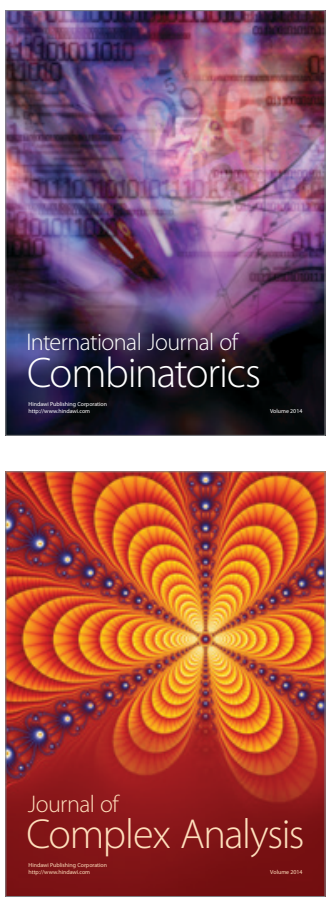

International Journal of

Mathematics and

Mathematical

Sciences
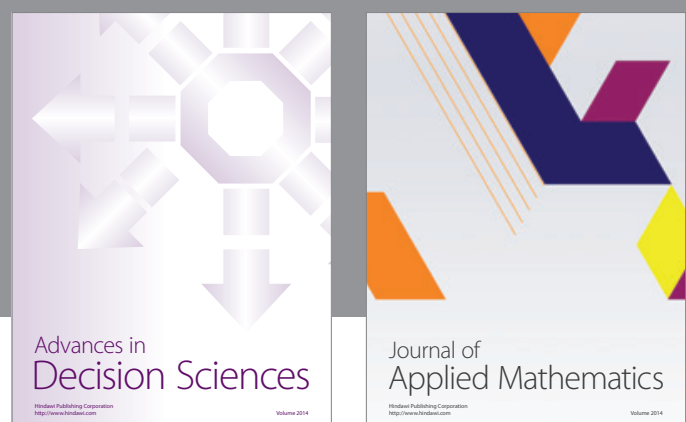

Journal of

Applied Mathematics
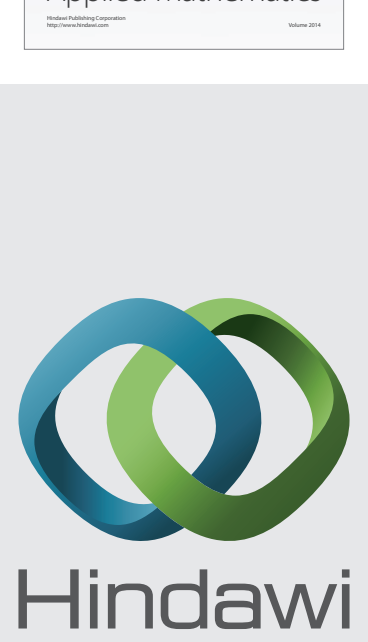

Submit your manuscripts at http://www.hindawi.com
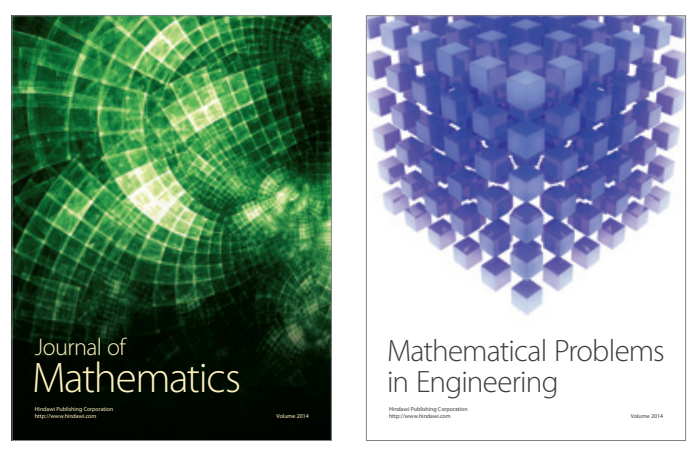

Mathematical Problems in Engineering
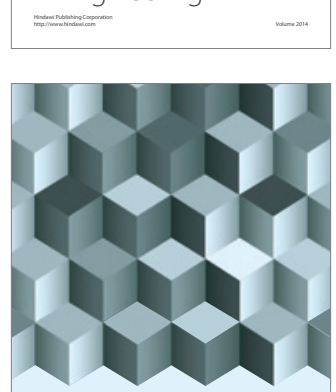

Journal of

Function Spaces
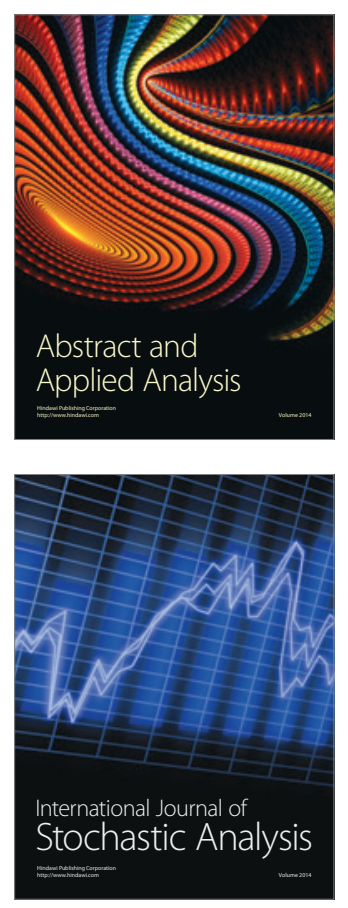

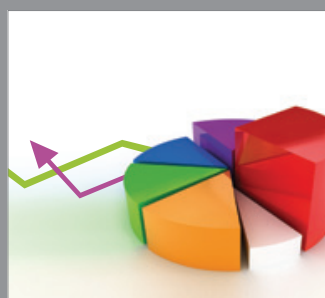

ournal of

Probability and Statistics

Promensencen
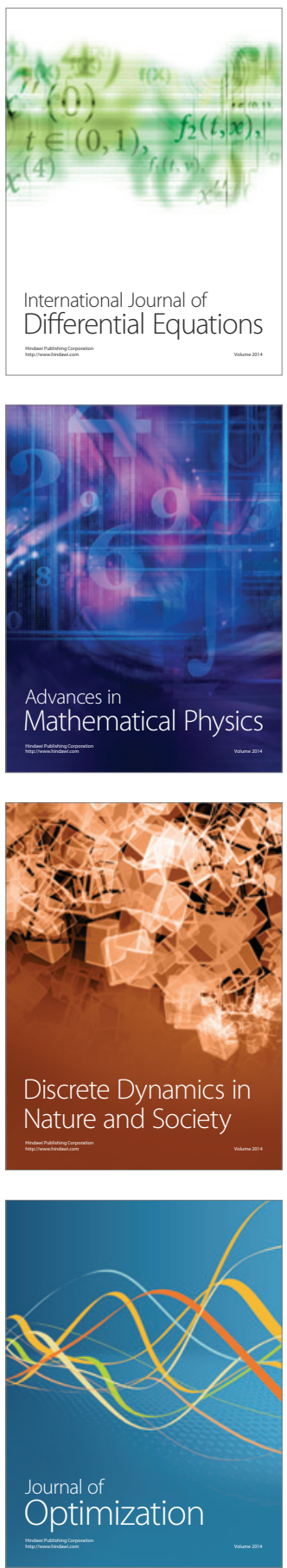